\title{
Technical Note: Estimation of Milk Fatty Acid Yield from Milk Fat Data
}

\author{
F. Glasser, ${ }^{1}$ M. Doreau, A. Ferlay, and Y. Chilliard \\ Institut National de la Recherche Agronomique (INRA), UR1213 Herbivores, Site de Theix, F-63122 Saint-Genès-Champanelle, France
}

\begin{abstract}
Most publications reporting milk fatty acid (FA) yields estimate these yields from milk fat yields and a coefficient estimating the proportion of FA in milk fat. The most widely used coefficient is, in fact, the proportion of fatty acyl radicals (i.e., FA from which the $\mathrm{OH}$ group has been removed) in milk triglycerides, equivalent to a mean proportion of $88 \%$ FA in milk fat. From a metabolic point of view, because the digestive flows and mammary FA uptake consist of nonesterified FA, it is more logical to estimate the FA proportion rather than the fatty acyl proportion in milk fat. From 588 milk FA profiles, we estimated a mean proportion of 94.4\% FA in milk triglycerides. Moreover, when the other milk lipid classes (phospholipids, diglycerides, etc.) were taken into account, the proportion of FA in milk total lipids was estimated at $93.3 \%$, almost independently of the milk FA profile. The use of this coefficient to estimate the secretion of milk FA on the basis of milk fat yield data is more physiologically relevant for milk FA secretion studies.
\end{abstract}

Key words: dairy cow, milk fat, fatty acid

In studies on the regulation of milk fat secretion, a common approach is to study the response of animals to experimental factors in terms of milk fatty acid (FA) yield (see recent examples in Loor et al., 2005; Corl et al., 2006). Daily milk yield and milk fat content are routinely measured, and milk fat yield can be computed from these 2 figures. Current methods for FA analysis are based on GLC analysis of FA methyl esters, producing data expressed as weight percentages of the various FA in a sample. Although total FA contents in feed, digesta, and plasma samples are determined directly by introducing a given FA in the sample as the internal standard (see, for example, Sukhija and Palmquist, 1988), no internal standard is generally used for milk sample analyses. Milk individual FA contents and yields must thus be estimated from the milk fat content

Received December 21, 2006.

Accepted January 16, 2007.

${ }^{1}$ Corresponding author: fglasser@clermont.inra.fr and yield and the FA proportions (generally expressed as weight percentages of total FA). As a consequence, to estimate the FA yield from the fat yield, the proportion of total FA in milk fat has to be estimated.

\section{The Current Method}

Most of the publications reporting milk FA yields (e.g., Christensen et al., 1998; Ruppert et al., 2003) use the estimation method published by Schauff et al. (1992). This method consists of

assuming that milk fat is $100 \%$ triglycerides and that there are $3 \mathrm{~mol}$ of fatty acids/mol of glycerol. Thus, $100 \mathrm{~mol}$ of fatty acids represent $33.3 \mathrm{~mol}$ of triglyceride. The weight that each of the fatty acids and glycerol contribute to the $33.3 \mathrm{~mol}$ of triglyceride was calculated after the molecular weight of each fatty acid was adjusted for removal of hydrogen and oxygen that are lost when the fatty acid is esterified to glycerol. The weights of the individual fatty acids and glycerol were converted to a relative weight percentage. The relative weight percentage was multiplied by yield of fat in milk to determine the yield of individual fatty acids and glycerol. (Schauff et al., 1992, p. 1925)

Schauff et al. (1992) removed the hydrogen and oxygen from the FA to compute the FA contribution to triglycerides (TG), which was, in fact, equivalent to estimating the proportion of fatty acyl radicals (i.e., FA from which the $\mathrm{OH}$ group had been removed) in TG, instead of that of FA in TG. We believe that this method leads to a systematic underestimation of milk FA yield. Moreover, because the resulting data are reported in publications as "fatty acid yields," it could lead to errors if a reader wanted to back-calculate yields in moles per day, for example, by applying FA molecular weights to these data expressed in grams per day.

Schauff et al. (1992) justified their method by the fact that hydrogen and oxygen are lost when the FA are esterified. Indeed, the esterification step is operated by mammary acyltransferases, which transfer fatty acyl radicals from coenzyme $\mathrm{A}$ to the glycerol backbone. 
More generally in esterification reactions, the oxygen in the ester comes from the alcohol.

However, this estimation method does not seem to be the most suitable one to use for metabolic or milk production studies. If we consider the fate of dietary lipids through the animal until milk secretion, the estimation of FA, and not fatty acyl radicals, seems to be more logical. Most of the time, dietary FA are esterified, mainly as TG or galactolipids. These esters are hydrolyzed to a large extent in the rumen, pass through the duodenum, and are absorbed as NEFA by the enterocytes. Absorbed NEFA are then esterified to be transported in the plasma. At the mammary level, they are hydrolyzed again by lipoprotein lipase, and absorbed as NEFA by the mammary gland, where they are finally esterified in milk lipids. Nonesterified FA originating from body reserve mobilization can also be taken up by the mammary gland. Thus, at the various metabolic levels (intestinal absorption, lipomobilization, mammary uptake), lipid flows consist of NEFA flows. For this reason, to compare the flows at the different levels, estimation of FA, rather than fatty acyl radicals, in milk TG seems to be a more logical method. Use of the proportion of fatty acyl radicals in milk fat would indeed induce a systematic bias between mammary FA uptake and fatty acyl secretion.

The same type of reasoning can be extended downstream in the food chain: Underestimating the FA content of milk fat by using the fatty acyl content figure could induce underestimation of the milk FA supply in human diets, for example (i.e., the FA that would be available after milk TG hydrolysis during digestion). For these reasons, we think that it is more pertinent to estimate the proportion of FA in milk fat rather than that of fatty acyl radicals.

\section{A New Proposal}

To compute the proportion of FA in milk TG, we propose a modification of the method of Schauff et al. (1992). In this new method, the oxygen does not have to be removed from the FA molecular weight. As a consequence, the "glycerol" moiety of the TG consists solely of the carbon backbone of the glycerol, because the oxygen atoms are considered as part of the FA, to be more consistent with metabolic FA flows (see above).

From 558 milk FA profiles compiled from published experiments (156 experiments published from 1967 to 2005), including cows fed low-lipid, plant oil-supplemented, and fish oil-supplemented diets, we estimated the FA proportion in milk TG. The mean molecular weight of milk FA in these data was $232 \mathrm{~g} / \mathrm{mol}$ (SD = $9 \mathrm{~g} / \mathrm{mol})$.
From an analytical point of view, we could compute the amount of FA that would be obtained by hydrolysis of a given amount of milk TG. Assuming a mean milk FA molecular weight of $232 \mathrm{~g} / \mathrm{mol}$, the mean milk TG has a molecular weight of $737 \mathrm{~g} / \mathrm{mol}$. Hydrolysis of 100 $\mathrm{g}$ of milk TG $(0.1357 \mathrm{~mol})$ would produce $0.1357 \mathrm{~mol}$ of glycerol $(=12.5 \mathrm{~g})$ plus $0.4071 \mathrm{~mol}(=3 \times 0.1337)$ of $\mathrm{FA}$ $(=94.4 \mathrm{~g})$. The amount of FA obtained from $100 \mathrm{~g}$ of milk TG would thus be $94.4 \mathrm{~g}$, which is equivalent to the necessary amount of precursor FA used by the mammary gland for TG synthesis.

From the 558 milk FA profiles, the method proposed in this paper produced a mean proportion of FA in milk TG of $94.4 \pm 0.2 \%$ (mean $\pm \mathrm{SD}$; i.e., a "glycerol" moiety of approximately $5.6 \%$ ), instead of $87.9 \% \pm 0.4 \%$ (i.e., glycerol approximately $12.1 \%$ ) by the method of Schauff et al. (1992). This last method thus leads to a systematic underestimation of milk FA yield by approximately $7 \%$.

The proportion of total FA in a given milk sample could thus be estimated from the fat content according to the following procedure: The weight percentages of the milk FA (the raw data most often obtained from GLC analyses) must first be converted to molar percentages. The mean molecular weight of the FA (MMWFA) can thus be computed as the mean of the individual FA molecular weights, weighted by their molar percentages. The mean TG consists of 3 mean FA molecules plus the carbon chain of glycerol $\left(\mathrm{C}_{3} \mathrm{H}_{5}\right.$, molecular weight of $41 \mathrm{~g} / \mathrm{mol}$ ), and the proportion of FA in milk TG (in percent) is obtained as

$$
3 \times \operatorname{MMWFA} /(3 \times \mathrm{MMWFA}+41) \times 100 .
$$

This coefficient, applied to milk fat content (or yield), gives an estimate of the milk total FA content (or yield). The individual FA figures (e.g., contents in grams per liter or yields in grams per day) can then be obtained using their weight percentages in total FA.

However, given the small variation in the coefficient obtained (with a mean of 94.4, more than $99 \%$ of the 558 published FA profiles had coefficients of between 94 and 95), we suggest that the coefficient of $94.4 \%$ could be applied uniformly to milk TG, whatever the FA profile. The resulting bias would be considerably lower than the other sources of variation in measurements during animal and laboratory experiments.

Bovine milk fat, however, contains other lipid constituents besides TG, namely, diglycerides (DG), monoglycerides, NEFA, cholesterol, cholesterol esters, and phospholipids (PL; Jensen, 2002). The proportions of FA in these molecules are different from the proportion of FA in TG. To estimate the proportion of FA in total milk lipids, we used the mean composition (or mean of the variation limits) of US milk provided by the National 
Dairy Council (cited by Jensen, 2002): 97.5\% TG, $0.435 \%$ DG, $0.6 \%$ PL, $0.27 \%$ NEFA, $0 \%$ cholesterol esters, $0.42 \%$ cholesterol, and $0.27 \%$ monoglycerides. These figures are very close to other published values (Bitman and Wood, 1990; Christie, 1995). The proportions of the different PL were set at (in mol \%): phosphatidylcholine, 34.5; phosphatidylethanolamine, 31.8; phosphatidylserine, 3.1; phosphatidylinositol, 4.7; and sphingomyelin, 25.2 (Jensen, 2002). We computed the proportion of FA in each lipid constituent and estimated the FA proportion in total milk lipids as the mean of these proportions weighted by the proportions of the constituents in total milk lipids. From the 558 milk FA profiles, the mean FA proportion in total milk fat was $93.3 \pm 0.2 \%$. Varying the proportions of the different lipid classes in milk fat had a very small effect on this figure: The proportions of Bitman and Wood (1990) with the smallest TG (95.8\%) and the highest PL (1.11\%) and DG $(2.25 \%)$ percentages gave a mean proportion of $93.4 \pm 0.2 \%$. Similarly, considering that the FA esterified on PL were all 18-carbon FA rather than shorter chain FA did not significantly modify the overall FA proportion in milk total lipids. The main source of variation of this coefficient was the FA proportion in milk TG (by far the most important constituent of milk fat), which is only slightly influenced by the milk FA profile, as stated above. As a consequence, just as with TG and given the low variability of the coefficient, we propose to apply a constant coefficient of $93.3 \%$ as the mean proportion of FA in milk total lipids, independently of the milk FA profile.

To conclude, to ensure consistency between FA flow measurements at various levels of animal metabolism, it seems more pertinent to compute milk FA yields from the proportion of FA in the milk TG, according to the method presented in this paper, rather than from the proportion of fatty acyl radicals, as used by most authors. Furthermore, taking the other milk lipid classes into account produces an estimate of $93.3 \%$ of $\mathrm{FA}$ in milk total lipids. The present approach may also be used to estimate the amounts of FA contained in other lipid sources, such as dietary TG from plant oils or seeds.

\section{REFERENCES}

Bitman, J., and D. L. Wood. 1990. Changes in milk fat phospholipids during lactation. J. Dairy Sci. 73:1208-1216.

Christensen, R. A., J. H. Clark, J. K. Drackley, and S. A. Blum. 1998. Fatty acid flow to the duodenum and in milk from cows fed diets that contained fat and nicotinic acid. J. Dairy Sci. 81:1078-1088.

Christie, W. W. 1995. Composition and structure of milk lipids. Pages 1-36 in Advanced Dairy Chemistry. Vol. 2, Lipids. P. F. Fox, ed. Chapman \& Hall, London, UK.

Corl, B. A., S. T. Butler, W. R. Butler, and D. E. Bauman. 2006. Short communication: Regulation of milk fat yield and fatty acid composition by insulin. J. Dairy Sci. 89:4172-4175.

Jensen, R. G. 2002. The composition of bovine milk lipids: January 1995 to December 2000. J. Dairy Sci. 85:295-350.

Loor, J. J., A. Ferlay, A. Ollier, M. Doreau, and Y. Chilliard. 2005. Relationship among trans and conjugated fatty acids and bovine milk fat yield due to dietary concentrate and linseed oil. J. Dairy Sci. 88:726-740.

Ruppert, L. D., J. K. Drackley, D. R. Bremmer, and J. H. Clark. 2003. Effects of tallow in diets based on corn silage or alfalfa silage on digestion and nutrient use by lactating dairy cows. J. Dairy Sci. 86:593-609.

Schauff, D. J., J. P. Elliott, J. H. Clark, and J. K. Drackley. 1992. Effects of feeding lactating dairy cows diets containing whole soybeans and tallow. J. Dairy Sci. 75:1923-1935.

Sukhija, P. S., and D. L. Palmquist. 1988. Rapid method for determination of total fatty acid content and composition of feedstuffs and feces. J. Agric. Food Chem. 36:1202-1206. 